\title{
New results on resource sharing problems with random agent arrivals and an application to economic dispatch in power systems
}

\author{
Alessandro Falsone, Kostas Margellos, Jacopo Zizzo, Maria Prandini, Simone Garatti
}

\begin{abstract}
We consider linear resource sharing problems with multiple agents. Agents are heterogeneous, with heterogeneity modelled by a tuple of parameters taking value according to an underlying probability distribution, and share a fixed resource amount. We provide an evaluation of a vital indicator for the correct operation of the agents, namely, the probability that the optimal resource share alters in case of a new agent arrival. We view this problem under a data driven lens, and provide a purely a-posteriori and prior-independent characterization of the above mentioned probability by exploiting recent developments in the so called scenario approach theory. The proposed framework is demonstrated on an economic dispatch example in power systems, where agents can be thought of as generating units participating in the power market.
\end{abstract}

\section{INTRODUCTION}

Resource sharing problems where multiple agents seek an optimal way to share a budget while satisfying their individual constraints appear in various engineering problems ranging from power systems [1], [2], to robotics and sensor networks [3], [4]. Despite the intense research activity in solving such problems in a computationally efficient manner by means of decentralized and distributed methodologies (see, e.g., [5]-[13]), a remaining challenge is assessing the sensitivity of the solution to a resource sharing problem when it comes to new agent arrivals, as discussed in [2] in the context of demand side management. The aim is determining the capacity of a multi-agent system in terms of the critical number of agents beyond which the arrival of a new agent does not have any effect on the share of resources which remains unaltered.

We focus on the particular class of multi-agent resource sharing problems that can be encoded by means of a linear program subject to equality budget constraints. This is a vast class, encompassing e.g. problems arising in the application domains outlined above, [1], [2], as well as the class of linear production processes that are encountered in cooperative game theory, [14], [15]. The amount of resource to be shared is fixed and agents are considered to be heterogeneous, with heterogeneity modelled by a tuple of parameters encompassing each agent's contribution to the linear cost and the constraints, and take values according to a fixed

Research was supported by the European Commission under the project UnCoVerCPS, grant number 643921, and by EPSRC UK under the grant $\mathrm{EP} / \mathrm{P} 03277 \mathrm{X} / 1$.

Alessandro Falsone, Jacopo Zizzo, Maria Prandini, and Simone Garatti are with the Dipartimento di Elettronica, Informazione e Bioingegneria, Politecnico di Milano, Via Ponzio 34/5, 20133 Milano, Italy (e-mail: name.surname@polimi.it, jacopo.zizzo@mail.polimi.it).

Kostas Margellos is with the Department of Engineering Science, University of Oxford, Parks Road, Oxford, OX1 3PJ, United Kingdom (e-mail: kostas.margellos@eng.ox.ac.uk). but possibly unknown multivariate probability distribution. A finite population of agents participating in the resource sharing problem is thus instantiated upon a multi-extraction of these parameters from the underlying distribution. Once the solution to the problem corresponding to a given agent population is determined, it might be that the arrival of a new agent either affects the computed optimal resource share, or leaves this unaffected. Since agents are random, in the sense that they are parameterized by a certain parameter tuple, the effect of a new agent arrival can only be probabilistically computed.

Preliminary results towards this direction were provided in [16], but without considering any upper bound on the agents' decision vectors determining their contribution to the resource sharing problem. Here, we extend these results by introducing local upper-limit constraints to be present in the resource sharing problem, with these limits constituting an additional random parameter that contributes to agents' heterogeneity. Accounting for these constraints when characterizing the probability that an optimal resource sharing solution remains unaltered upon a new agent arrival is, however, far from being trivial and requires a new theoretical analysis line.

To address this problem, we view it under a data driven lens and provide a confidence interval for a probabilistic sensitivity index given by the probability that the optimal resource share changes upon the arrival of a new agent. Such a result is based on a combination of tools from linear programming, duality theory, and the so called scenario approach, [17], [18], [19], especially the scenario approach with constraint relaxation, [20], [21]. However, the standard scenario approach theory is based on the existence of a certain subset of the samples with an a priori fixed cardinality that produces the same solution that is obtained when all the samples are employed. The presence of upper-limit constraints in the resource sharing problem considered in this paper results in this cardinality to be increasing with the number of agents (which corresponds to uncertainty scenarios in our context), hence making the standard scenario approach theory inapplicable. To this end we move away from the a priori analysis that was pursued in [16], and rather follow an a posteriori route, exploiting the recent "wait-andjudge" developments of the scenario approach, [22], [23], [20]. This allows us to obtain a tight quantification of the probability that the optimal resource share changes upon the arrival of a new agent based on the value taken by an observable and easy to compute quantity, which is the number of agents that are actually utilized in the current 
solution.

The proposed framework is demonstrated on an economic dispatch example in power systems. This entails dispatching the generating units so that a given load is served at the minimum cost. Such a problem directly fits in the proposed setting, where generating units play the role of agents participating in the power market and the load plays the role of the resource to be shared.

The remainder of the paper unfolds as follows. Section II states the resource sharing program under study, while Section III states our main result, i.e., the provision of upper and lower bounds on the probability that the solution to the resource sharing problem under study changes upon the arrival of a new agent. Section IV provides a detailed application of our methodology to the problem of economic dispatch in power systems. Section V concludes the paper and provides directions for future work. Proofs for all mathematical statements can be found in [24].

\section{Mathematical FRAMEWORK}

The multi-agent optimal resource sharing problems considered in this paper are as follows:

$$
\begin{aligned}
& \mathcal{P}^{m}: \min _{\left\{x^{i} \in \mathbb{R}^{n^{i}}\right\}_{i=1}^{m}} \sum_{i=1}^{m}\left(c^{i}\right)^{\top} x^{i} \\
& \text { subject to: } x^{i} \geq 0, i=1, \ldots, m \text {, } \\
& \sum_{i=1}^{m} A^{i} x^{i}=b, \\
& x^{i} \leq d^{i}, i=1, \ldots, m,
\end{aligned}
$$

where $m \in \mathbb{N}_{+}$denotes the number of agents. For all $i=$ $1, \ldots, m$, let $c^{i} \in \mathbb{R}^{n^{i}}, d^{i} \in \mathbb{R}^{n^{i}}$ and $A^{i} \in \mathbb{R}^{p \times n^{i}}$. Moreover, for all $i=1, \ldots, m, x^{i} \in \mathbb{R}^{n^{i}}$ denotes the vector of decision variables associated to agent $i$, while $b \in \mathbb{R}^{p}$ denotes the total amount of $p$ different resources to be shared among agents. Row $r$ of each $A^{i}$ returns the consumption of the $r$ th resource corresponding to a unitary value of the decision variables $x^{i}$ of agent $i ;\left(c^{i}\right)^{\top} x^{i}$ is the cost associated to $x^{i}$, while $d^{i}$ is an upper limit imposed to the value that can be taken by $x^{i}$.

Problem $\mathcal{P}^{m}$ then allocates the resources to various agents by instantiating their decision variables so as to minimize the global cost while satisfying the constraints. These are: the non-negativity constraints $x^{i} \geq 0$, the budget type constraint $\sum_{i=1}^{m} A^{i} x^{i}=b$, and the upper-limit constraints $x^{i} \leq d^{i}$, which are all meant component-wise. Note that, possibly, $n^{i} \neq n^{j}$ for $i \neq j$.

Note that $\mathcal{P}^{m}$ in (1) is not a linear program in standard form, [25], due to the presence of the upper-limit constraints. It could be brought to standard form via the introduction of additional slack variables, [25, Section 1.1]; however, the number of coupling budget-type constraints would then be proportional to the number of agents $m$. We prefer to show the upper-limit constraints explicitly since this offers additional interpretation to our results. Moreover, note that the budget constraints are assumed to be equalities; the case of inequality constraints can be captured by introducing additional slack variables as performed in [24].

In $\mathcal{P}^{m}$ each agent $i, i=1, \ldots, m$, is fully characterized by the tuple $\delta^{i}=\left(n^{i}, c^{i}, d^{i}, A^{i}\right)$. Here, we assume that $\delta^{i}$, $i=1, \ldots, m$, are independent and identically distributed (i.i.d.) samples of a random quantity $\delta=(n, c, d, A)$ defined over a generic probability space $(\Delta, \mathcal{D}, \mathbb{P})$. As $(\Delta, \mathcal{D}, \mathbb{P})$ models the unknown population from which agents arise, $\mathbb{P}$ is supposed to be unknown too. It should be noted that $\mathbb{P}$ corresponds to the joint probability distribution of the elements of $(n, c, d, A)$; in the particular case where all agents have decision vectors of the same length, then the marginal probability of $n$ will be concentrated to that value. Given the i.i.d. assumption, the collection $\left\{\delta^{i}\right\}_{i=1}^{m}$ is distributed according to the product measure $\mathbb{P}^{m}$.

Under this setting, $\mathcal{P}^{m}$ becomes a random linear program, with the number of agents corresponding to the number of realizations of the uncertain tuple $(n, c, d, A)$ that have instantiated $\mathcal{P}^{m}$. We impose the following assumption.

Assumption 1 (Feasibility and uniqueness): For any $m \in \mathbb{N}_{+}$, the linear program $\mathcal{P}^{m}$ in (1) is feasible and admits a unique minimizer almost surely with respect to $\mathbb{P}^{m}$.

\section{Characterization OF The Sensitivity of The SOLUTION TO THE ARRIVAL OF A NEW AGENT}

Suppose that a new agent characterized by $\bar{\delta}=(\bar{n}, \bar{c}, \bar{d}, \bar{A})$ joins the resource sharing problem, and let $\bar{x} \in \mathbb{R}^{\bar{n}}$ denote its corresponding decision vector. The resulting linear program $\mathcal{P}_{+}^{m}$ for the $(m+1)$-agent problem is as follows:

$$
\begin{aligned}
& \mathcal{P}_{+}^{m}: \min _{\left\{x^{i} \in \mathbb{R}^{n^{i}}\right\}_{i=1}^{m}, \bar{x} \in \mathbb{R}^{\bar{n}}} \sum_{i=1}^{m}\left(c^{i}\right)^{\top} x^{i}+\bar{c}^{\top} \bar{x} \\
& \text { subject to: } \quad x^{i} \geq 0, i=1, \ldots, m, \bar{x} \geq 0, \\
& \sum_{i=1}^{m} A^{i} x^{i}+\bar{A} \bar{x}=b, \\
& x^{i} \leq d^{i}, i=1, \ldots, m, \bar{x} \leq \bar{d} .
\end{aligned}
$$

Let $x=\left[\left(x^{1}\right)^{\top} \ldots\left(x^{m}\right)^{\top}\right]^{\top} \in \mathbb{R}^{\ell}$, with $\ell=\sum_{i=1}^{m} n^{i}$, be the vector of all decision variables of all agents in $\mathcal{P}^{m}$ in (1). Analogously, $x_{+}=\left[x^{\top} \bar{x}^{\top}\right]^{\top} \in \mathbb{R}^{\ell+\bar{n}}$ is the vector of the decision variables of $\mathcal{P}_{+}^{m}$ in (2). The optimal solutions to $\mathcal{P}^{m}$ and $\mathcal{P}_{+}^{m}$ are denoted by $x^{\star}$ and $x_{+}^{\circ}=\left[\left(x^{\circ}\right)^{\top}\left(\bar{x}^{\circ}\right)^{\top}\right]^{\top}$, respectively. Thanks to Assumption $1, x^{\star}$ and $x_{+}^{\circ}$ are almost surely, with respect to $\mathbb{P}^{m+1}$, uniquely defined.

The optimal solution to $\mathcal{P}_{+}^{m}$ in (2) can either be such that: (a) $\bar{x}^{\circ}=0$, in which case we must have $x_{+}^{\circ}=\left[\left(x^{\star}\right)^{\top} 0^{\top}\right]$ because, otherwise, if $x_{+}^{\circ}=\left[\left(x^{\circ}\right)^{\top} 0^{\top}\right] \neq\left[\left(x^{\star}\right)^{\top} 0^{\top}\right]$, then $x^{\circ}$ would achieve a strictly smaller cost for $\mathcal{P}^{m}$ in (1) than its optimal solution $x^{\star}$, or (b) $\bar{x}^{\circ} \neq 0$ and $x_{+}^{\circ} \neq\left[\left(x^{\star}\right)^{\top} 0^{\top}\right]$, in which case we have that the optimal value of $\mathcal{P}_{+}^{m}$ improves over that of $\mathcal{P}^{m}$ because $\left[\left(x^{\star}\right)^{\top} 0^{\top}\right]$ is always feasible for $\mathcal{P}_{+}^{m}$. 
For a resource sharing problem with $m$ agents, our objective is to quantify how likely it is that the arrival of a new agent improves the optimal solution achieved by the initial $m$ agents alone. More formally, given that the new agent is characterized by a stochastic tuple $\bar{\delta}=(\bar{n}, \bar{c}, \bar{d}, \bar{A})$, we are interested in quantifying the probability with which $x_{+}^{\circ} \neq\left(x^{\star}, 0\right)$, i.e., $\mathbb{P}\left\{\bar{\delta}: x_{+}^{\circ} \neq\left(x^{\star}, 0\right)\right\}$, which then serves as a probabilistic sensitivity index. It must be noticed, however, that $\mathbb{P}\left\{\bar{\delta}: x_{+}^{\circ} \neq\left(x^{\star}, 0\right)\right\}$ cannot be directly computed, since $\mathbb{P}$ is not known (remember that $\mathbb{P}$ models an unknown population). The evaluation of $\mathbb{P}\left\{\bar{\delta}: x_{+}^{\circ} \neq\right.$ $\left.\left(x^{\star}, 0\right)\right\}$ therefore must proceed along a different route.

To this purpose, observe that, by considering the dependence (which is not explicitly shown to avoid notational clutter) of $x^{\star}$ and $x_{+}^{\circ}$ on the random sample $\left\{\delta^{i}\right\}_{i=1}^{m}$, the quantity of interest $\mathbb{P}\left\{\bar{\delta}: x_{+}^{\circ} \neq\left(x^{\star}, 0\right)\right\}$ can be regarded as a random variable defined over the product probability space $\left(\Delta^{m}, \mathcal{D}^{m}, \mathbb{P}^{m}\right)$. Hence, we will provide upper and lower bounds on $\mathbb{P}\left\{\bar{\delta}: x_{+}^{\circ} \neq\left(x^{\star}, 0\right)\right\}$, that will hold with confidence measured with respect to $\mathbb{P}^{m}$. We impose in addition the following assumption.

Assumption 2 (Non-degeneracy): We assume that for any $m \in \mathbb{N}_{+}$:

1) For all $i=1, \ldots, m, d^{i}>0$.

2) At any feasible point for $\mathcal{P}^{m}$ in (1), no more than $\ell$ constraints are active almost surely.

3) For any vector $\lambda \in \mathbb{R}^{p}$,

$$
\begin{array}{r}
\mathbb{P}\{\delta=(n, c, d, A) \in \Delta: \exists j \in\{1, \ldots, n\} \\
\text { such that } \left.\left[c^{\top}+\lambda^{\top} A\right]_{j}=0\right\}=0,
\end{array}
$$

where $[\cdot]_{j}$ denotes the $j$-th element of its argument.

Assumption 2 imposes certain non-degeneracy conditions. In particular, part 1 excludes the case of degenerate agents with $x^{i}$ being forced to be equal to zero. Part 2 implies that $\mathcal{P}^{m}$ in (1) is non-degenerate in the sense of [25, Definition 2.10]. The condition of part 3 implies that for any given $\lambda \in \mathbb{R}^{p}$, the probability that $\lambda$ belongs to the boundary of the affine constraints $c^{\top}+\lambda^{\top} A \leq 0$ is zero. In other words, these affine constraints, parameterized by the elements $c$ and $A$ of $\delta$, do not accumulate over the same point at their boundaries with the exception of zero probability cases only. Such a condition appears also in [20], and is invoked in the proof of Theorem 1. Both conditions 2 and 3 in Assumption 2 are quite mild provided that $\delta=(n, c, d, A)$ is generically distributed with no concentrated mass in the marginal distributions of $c, d$, and $A$.

Fix now any $\beta \in(0,1)$ and for any $k=0,1, \ldots, m-1$, consider the following polynomial equation in the variable $t$ (see [20, Theorem 4]),

$$
\begin{aligned}
\left(\begin{array}{c}
m \\
k
\end{array}\right) t^{m-k}-\frac{\beta}{2 m} \sum_{i=k}^{m-1}\left(\begin{array}{l}
i \\
k
\end{array}\right) t^{i-k} & \\
& -\frac{\beta}{6 m} \sum_{i=m+1}^{4 m}\left(\begin{array}{l}
i \\
k
\end{array}\right) t^{i-k}=0
\end{aligned}
$$

and for $k=m$ consider the polynomial equation

$$
1-\frac{\beta}{6 m} \sum_{i=m+1}^{4 m}\left(\begin{array}{l}
i \\
k
\end{array}\right) t^{i-m}=0 .
$$

As shown in [20], for any $k=0,1, \ldots, m-1$, (4) has exactly two solutions denoted as $\underline{t}(k), \bar{t}(k) \in[0,+\infty)$, with $\underline{t}(k) \leq \bar{t}(k)$, while (5) has only one solution denoted by $\bar{t}(m) \in[0,+\infty) ;{ }^{1}$ we also define $\underline{t}(m)=0$. Define then the functions $\underline{\epsilon}(\cdot), \bar{\epsilon}(\cdot):\{0,1, \ldots, m\} \rightarrow[0,1]$ as

$$
\begin{aligned}
& \underline{\epsilon}(k)=\max \{0,1-\bar{t}(k)\}, \\
& \bar{\epsilon}(k)=\max \{0,1-\underline{t}(k)\},
\end{aligned}
$$

$k=0,1, \ldots, m$. We are now ready to state the main result of our paper.

Theorem 1: Consider Assumptions 1 and 2. Fix $\beta \in$ $(0,1)$, and consider $\underline{\epsilon}(\cdot)$ and $\bar{\epsilon}(\cdot)$ as defined in (6) and (7), respectively. Denote then by $s^{\star}$ the number of agents whose decision vector has at least one non-zero element, i.e.,

$$
\begin{aligned}
s^{\star}=\mid\{i & \in\{1, \ldots, m\}: \\
& \left.\exists j \in\left\{1, \ldots, n^{i}\right\} \text { such that } x_{j}^{i, \star} \neq 0\right\} \mid,
\end{aligned}
$$

where $|\cdot|$ denotes the cardinality of its argument and $x_{j}^{i, \star}$ denotes the $j$-th element of the $i$-th component of the optimal solution $x^{\star}$. We then have that

$$
\begin{aligned}
& \mathbb{P}^{m}\left\{\left\{\delta^{i}\right\}_{i=1}^{m} \in \Delta^{m}:\right. \\
& \left.\quad \mathbb{P}\left\{\bar{\delta} \in \Delta: x_{+}^{\circ} \neq\left(x^{\star}, 0\right)\right\} \in\left[\underline{\epsilon}\left(s^{\star}\right), \bar{\epsilon}\left(s^{\star}\right)\right]\right\} \geq 1-\beta .
\end{aligned}
$$

\section{Proof of Theorem 1: see [24].}

Theorem 1 implies that the probability that the optimal solution $x^{\star}$ of $\mathcal{P}^{m}$ in (1) changes upon the arrival of a new agent lies within the interval $\left[\underline{\epsilon}\left(s^{\star}\right), \bar{\epsilon}\left(s^{\star}\right)\right]$ with confidence at least $1-\beta$. The quantity $s^{\star}$ that appears in the interval definition is itself a random variable, since it depends on the random sample $\left\{\delta^{i}\right\}_{i=1}^{m}$, and is always highly correlated with $\mathbb{P}\left\{\bar{\delta}: x_{+}^{\circ} \neq\left(x^{\star}, 0\right)\right\}$. However, unlike the quantity of interest $\mathbb{P}\left\{\bar{\delta} \in \Delta: x_{+}^{\circ} \neq\left(x^{\star}, 0\right)\right\}, s^{\star}$ is a computable one since it can be a posteriori determined by a direct inspection of $x^{\star}$. The essential message conveyed by Theorem 1 is that the observable $\left[\underline{\epsilon}\left(s^{\star}\right), \bar{\epsilon}\left(s^{\star}\right)\right]$ always provides a correct quantification (with confidence $1-\beta$ ) of the sought but unknown quantity $\mathbb{P}\left\{\bar{\delta} \in \Delta: x_{\delta}^{\star} \neq\left(x^{\star}, 0\right)\right\}$.

This quantification is often tight since, as shown in [20] and [26], $\underline{\epsilon}(k)$ and $\bar{\epsilon}(k)$ rapidly get close each other as $m$ increases, while their value is barely affected by $\beta$, so that very small values like $\beta=10^{-6}$ or $\beta=10^{-8}$ can be enforced to obtain that $\mathbb{P}\left\{\bar{\delta} \in \Delta: x_{+}^{\circ} \neq\left(x^{\star}, 0\right)\right\} \in\left[\underline{\epsilon}\left(s^{\star}\right), \bar{\epsilon}\left(s^{\star}\right)\right]$ with practical certainty. This can be also appreciated in Figure 1, which depicts $\underline{\epsilon}(k)$ and $\bar{\epsilon}(k)$ for $\beta=10^{-6}$ and various values for $m$.

\footnotetext{
${ }^{1}$ The solutions to (4) and (5) can be easily found by means of a bisection numerical algorithm; see [20, Appendix A] for a MATLAB implementation.
} 


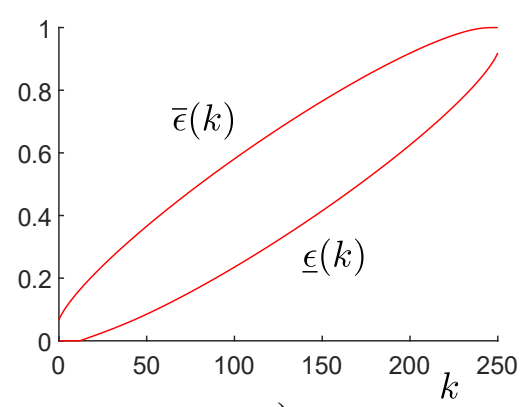

a)

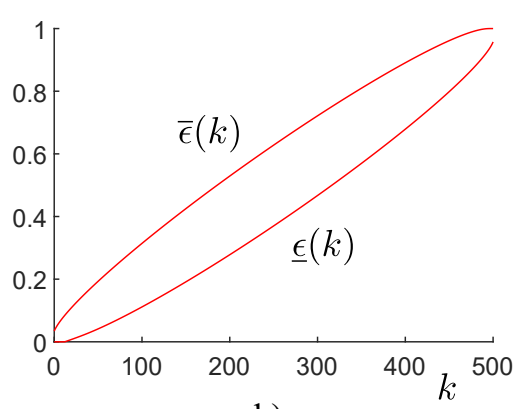

b)

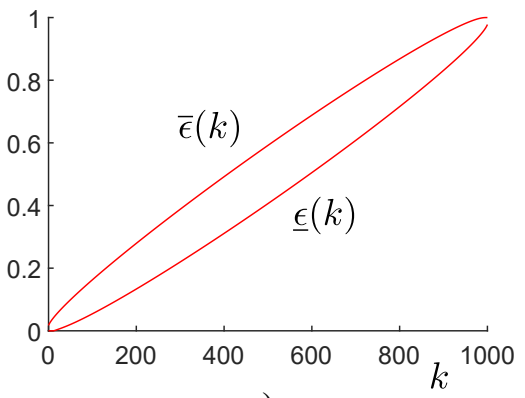

c)

Fig. 1. $\underline{\epsilon}(k)$ and $\bar{\epsilon}(k)$ for $\beta=10^{-6}$ and: a) $m=250$; b) $m=500$; c) $m=1000$.

Eventually, it is worth noticing that the result of Theorem 1 holds irrespective of the probability measure $\mathbb{P}$ (as long as Assumption 2 is satisfied), hence irrespective of the distribution of the agents' population.

\section{APPLICATION TO ECONOMIC DISPATCH PROBLEM}

Ever-rising costs of fossil fuels and the need for a better usage of renewable sources of energy for a suistainable development have made power system planning ever more pressing in energy production. The economic dispatch problem is a planning procedure carried out by a dispatch company that has to meet a (typically forecasted) load by several energy producers in a given geographical area. It involves determining the optimal power output from available energy generation facilities so as to minimize the overall production cost while meeting the required load and complying with power limits of each facility. If we denote with $P^{i}$ the power produced by the $i$-th facility, with $\mathcal{C}^{i}\left(P^{i}\right)$ the associated production cost, and with $L$ the load energy request, then the (simplified) energy dispatch problem considered in this paper is formalized as

$$
\begin{array}{ll}
\min _{\left\{P^{i} \in \mathbb{R}\right\}_{i=1}^{m}} & \sum_{i=1}^{m} \mathcal{C}^{i}\left(P^{i}\right) \\
\text { subject to: } & \sum_{i=1}^{m} P^{i}=L, \\
& 0 \leq P^{i} \leq P_{\max }^{i}, \quad i=1, \ldots, m,
\end{array}
$$

where each cost function $\mathcal{C}^{i}(\cdot)$ is convex, increasing, and such that $\mathcal{C}^{i}(0)=0$. Note that the overall production is required to exactly match the load $L$ (as opposed to be greater than or equal to it) because storing excess energy would be extremely costly and viable only for a limited amount of energy. Clearly, it must be $L \leq \sum_{i=1}^{m} P_{\max }^{i}$ for (8) to be feasible.

Even if $\mathcal{C}^{i}(\cdot)$ is in general a nonlinear function, it is often the case that $\mathcal{C}^{i}(\cdot)$ can be well approximated by a PieceWise Affine (PWA) function. To this end, for each generating facility $i$, we split the interval $\left[0, P_{\max }^{i}\right]$ into $n^{i}$ parts $\left[\bar{P}_{0}^{i}, \bar{P}_{1}^{i}\right]$, $\left[\bar{P}_{1}^{i}, \bar{P}_{2}^{i}\right], \ldots,\left[\bar{P}_{n^{i}-1}^{i}, \bar{P}_{n^{i}}^{i}\right]$, with $\bar{P}_{0}^{i}=0$ and $\bar{P}_{n^{i}}^{i}=P_{\max }^{i}$, and we assign a cost coefficient $c_{j}^{i}$ to the segment $\left[\bar{P}_{j-1}^{i}, \bar{P}_{j}^{i}\right]$, for all $j=1, \ldots, n^{i}$. Since the per unit production cost is increasing with $P^{i}$, it holds that $c_{1}^{i}<c_{2}^{i}<\cdots<c_{n^{i}}^{i}$, for all $i=1, \ldots, m$. If we denote with $x_{j}^{i}$ the amount of power produced within the segment $\left[\bar{P}_{j-1}^{i}, \bar{P}_{j}^{i}\right]$, then we can express $P^{i}=\sum_{j=1}^{n^{i}} x_{j}^{i}$ with $0 \leq x_{j}^{i} \leq d_{j}^{i}=\bar{P}_{j}^{i}-\bar{P}_{j-1}^{i}$, and rewrite (8) as

$$
\begin{array}{ll}
\min _{\left\{x^{i} \in \mathbb{R}^{n^{i}}\right\}_{i=1}^{m}} & \sum_{i=1}^{m}\left(c^{i}\right)^{\top} x^{i} \\
\text { subject to: } & \sum_{i=1}^{m} \mathbf{1}^{\top} x^{i}=L, \\
& 0 \leq x^{i} \leq d^{i}, \quad i=1, \ldots, m,
\end{array}
$$

where $x^{i}=\left[x_{1}^{i} \cdots x_{n^{i}}^{i}\right]^{\top}, c^{i}=\left[c_{1}^{i} \cdots c_{n^{i}}^{i}\right]^{\top}, d^{i}=$ $\left[d_{1}^{i} \cdots d_{n^{i}}^{i}\right]^{\top}$, and $\mathbf{1}=[1 \cdots 1]^{\top} \in \mathbb{R}^{n^{i}}$. It is easy to see that problem (9) fits the structure of $\mathcal{P}^{m}$ in (1), setting $A^{i}=\mathbf{1}^{\top}$ and $b=L$. Note also that the solution to (9) is such that, for each $i$ and for all $j=1, \ldots, n^{i}-1, x_{j+1}^{i}>0$ only if $x_{j}^{i}=d_{j}^{i}$, because the $c_{j}^{i}$ are increasing with $j$. This means that no power will be allocated in the interval $\left[\bar{P}_{j}^{i}, \bar{P}_{j+1}^{i}\right]$ if the previous interval $\left[\bar{P}_{j-1}^{i}, \bar{P}_{j}^{i}\right]$ has not been fully exploited, thus making $\left(c^{i}\right)^{\top} x^{i}$ an actual PWA approximation of $\mathcal{C}^{i}\left(P_{i}\right)$ for $P_{i}=\mathbf{1}^{\top} x^{i}$.

Suppose now that, by solving (9), the energy dispatch company has successfully planned the optimal usage of a population of known power plants in order to meet the required load. The company is certainly interested in reducing the costs by searching for additional facilities able to supply the desired area that may have smaller costs or better capacity. However, finding new facilities is a costly and time consuming operation, so it would be ideal for the company knowing in advance how beneficial this poll for new generators could be. The framework proposed in this paper can be used in order to retrieve the probability that this polling operation could bring a change in the original solution: a high probability of improving the solution will act as an incentive to start a new search for better facilities; otherwise, the dispatch company will stick with the solution found.

The availability of a new generating facility in the energy 
dispatch problem yields the optimization problem

$$
\begin{aligned}
\min _{\substack{\left\{x^{i} \in \mathbb{R}^{n^{i}}\right\}_{i=1}^{m}, \mathbb{R}^{n} \\
\text { subject to: }}} & \sum_{i=1}^{m}\left(c^{i}\right)^{\top} x^{i}+\bar{c}^{\top} \bar{x} \\
& \mathbf{1}^{\top} x^{i}+\mathbf{1}^{\top} \bar{x}=L, \\
& 0 \leq x^{i} \leq d^{i}, \quad i=1, \ldots, m, \\
& 0 \leq \bar{x} \leq \bar{d},
\end{aligned}
$$

and Theorem 1 ensures that a valid with confidence $1-\beta$ assessment of the probability that the solution to (10) improves over that of (9) is given by the interval $\left[\underline{\epsilon}\left(s^{\star}\right), \bar{\epsilon}\left(s^{\star}\right)\right]$, where $\underline{\epsilon}$ and $\bar{\epsilon}$ are computed as in (6)-(7) and $s^{\star}$ is the number of non zero components in the optimal solution to (9).

To test numerically the validity of Theorem 1, problem (9) was repeatedly solved 100 times with different batches of $m$ production facilities, and each time the optimal solution $x_{(t)}^{\star}$, $t=1, \ldots, 100$, and $s_{(t)}^{\star}, t=1, \ldots, 100$, were computed. For each $x_{(t)}^{\star}, M=50 \cdot m$ new generating plants corresponding to various $\bar{n}, \bar{d}, \bar{c}$ were then considered and problem (10) was solved $M$ times so as to compute

$$
\hat{\mathbb{P}}\left\{x_{+,(t)}^{\circ} \neq\left(x_{(t)}^{\star}, 0\right)\right\}=\frac{\text { no. of cases s.t. } x_{+,(t)}^{\circ} \neq\left(x_{(t)}^{\star}, 0\right)}{M},
$$

which is an empirical evaluation of the probability that the solution $x_{+,(t)}^{\circ}$ to (10) improves over $x_{(t)}^{\star}$. The pairs $\left(s_{(t)}^{\star}, \hat{\mathbb{P}}\left\{x_{+,(t)}^{\circ} \neq\left(x_{(t)}^{\star}, 0\right)\right\}\right)$ were then plotted in a bidimensional graph along with the curves $\underline{\epsilon}(k)$ and $\bar{\epsilon}(k)$ so as to allow one for a visual inspection that $\hat{\mathbb{P}}\left\{x_{+,(t)}^{\circ} \neq\left(x_{(t)}^{\star}, 0\right)\right\}$ is indeed within $\left[\underline{\epsilon}\left(s_{(t)}^{\star}\right), \bar{\epsilon}\left(s_{(t)}^{\star}\right)\right]$ as predicted by Theorem 1 .

We performed eight simulation campaigns: four with $m=$ 100 and four with $m=200$ for the number of agents. For each value for $m$, each energy producer $i$ was generated as follows:

- $n^{i}$ was independently extracted from a uniform distribution over the integers $3,4, \ldots, 10$;

- $P_{\max }^{i}$ was extracted from a uniform distribution over $\left[P_{\min }, P_{\max }\right]$, with $P_{\min }=100$ and we let $P_{\max } \in$ $\{200,400,800,1600\}$ (the four values of $P_{\max }$ correspond to the four test campaigns with the same value of $m$ );

- $\bar{P}_{0}^{i}, \ldots, \bar{P}_{n_{i}}^{i}$ were extracted from a uniform distribution over $\left[P_{\min }, P_{\max }^{i}\right]$ and then ordered;

- $c_{1}^{i}, \ldots, c_{n_{i}}^{i}$ were extracted from a uniform distribution over $[0,5]$ and then ordered.

In all tests the confidence parameter was set to $\beta=10^{-7}$. Figure $2 \mathrm{a}$ depicts the results obtained for $m=100$ and different values of $P_{\max }$, as reported in the figure legend. As $P_{\max }$ changes, different clouds of points are obtained corresponding to various batches of energy producers. In all cases, as predicted by Theorem $1, \hat{\mathbb{P}}\left\{x_{+,(t)}^{\circ} \neq\left(x_{(t)}^{\star}, 0\right)\right\}$ is in between $\underline{\epsilon}\left(s^{\star}\right)$ and $\bar{\epsilon}\left(s^{\star}\right)$ (given that $\beta=10^{-7}, \hat{\mathbb{P}}\left\{x_{+,(t)}^{\circ} \neq\right.$ $\left.\left(x_{(t)}^{\star}, 0\right)\right\} \notin\left[\underline{\epsilon}\left(s_{(t)}^{\star}\right), \bar{\epsilon}\left(s_{(t)}^{\star}\right)\right]$ should occur in one case out of 10 billions on average). From the figure, it is apparent that $\hat{\mathbb{P}}\left\{x_{+,(t)}^{\circ} \neq\left(x_{(t)}^{\star}, 0\right)\right\}$ gets smaller as $P_{\max }$ grows. Indeed, for large values of $P_{\max }$ it is more likely to find a producer that can cover a large amount of the load $L$ so that the number of employed producers tends to be moderate.

Similar comments apply to Figure $2 b$, where the results for $m=200$ and the same values for $P_{\max }$ as before are displayed. Notice that, as the number $m$ of agents increases, $\underline{\epsilon}(k)$ and $\bar{\epsilon}(k)$ approach each other, resulting in a tighter assessment of $\hat{\mathbb{P}}\left\{x_{+,(t)}^{\circ} \neq\left(x_{(t)}^{\star}, 0\right)\right\}$. Coherently, the clouds of points have smaller vertical dispersion in these simulations.

\section{CONCLUDING REMARKS}

In this paper we considered the class of multi-agent, linear resource sharing problems, where the amount of resource to be shared is fixed, and agents are subject to budget equality constraints as well as local limits. Agents are heterogeneous, and heterogeneity is modelled by means of a tuple of parameters involving agents' contributions to both the cost and the constraints. These parameters follow a fixed but possibly unknown multivariate probability distribution, and the resource sharing problem is instantiated upon a multiextraction of these parameters.

In this context, we provided a confidence interval for a probabilistic sensitivity index of the solution to the arrival of a new agent, namely, we provided upper and lower bounds for the probability that the optimal solution to the resource sharing problem changes upon the arrival of a new agent. Our bounds are a posteriori in the vein of the recent "wait-and-judge" developments of the scenario approach. The appealing feature of the proposed bounds is that they can be determined by counting the number of agents that are actually contributing to the solution of the original problem, and are independent of all other problem data. The efficacy of our results was demonstrated on an economic dispatch example in power systems, where agents were treated as generating units participating in the power market. Current work concentrates towards extending the class of resource sharing programs to more general convex optimization problems.

\section{REFERENCES}

[1] J. Warrington, P. Goulart, S. Mariéthoz, and M. Morari, "Policy-based reserves for power systems," IEEE Transactions on Power Systems, vol. 28 , no. 4 , pp. $4427-4437,2013$.

[2] K. Margellos and S. Oren, "Capacity controlled demand side management: A stochastic pricing analysis," IEEE Transactions on Power Systems, vol. 31, no. 1, pp. 706-717, 2016.

[3] S. Martínez, F. Bullo, J. Cortés, and E. Frazzoli, "On synchronous robotic networks - Part i: Models, tasks, and complexity," IEEE Transactions on Automatic Control, vol. 52, pp. 2199-2213, 2007.

[4] M. Stanković, K. Johansson, and D. Stipanović, "Distributed seeking of Nash equilibria with applications to mobile sensor networks," IEEE Transactions on Automatic Control, vol. 57, no. 4, pp. 904-919, 2012.

[5] D. Bertsekas and J. Tsitsiklis, Parallel and distributed computation: Numerical methods. Athena Scientific (republished in 1997), 1989.

[6] A. Nedić and A. Ozdaglar, "Distributed subgradient methods for multiagent optimization," IEEE Transactions on Automatic Control, vol. 54, no. 1, pp. 48-61, 2009.

[7] A. Nedić, A. Ozdaglar, and P. Parrillo, "Constrained consensus and optimization in multi-agent networks," IEEE Transactions on Automatic Control, vol. 55, no. 4, pp. 922-938, 2010. 


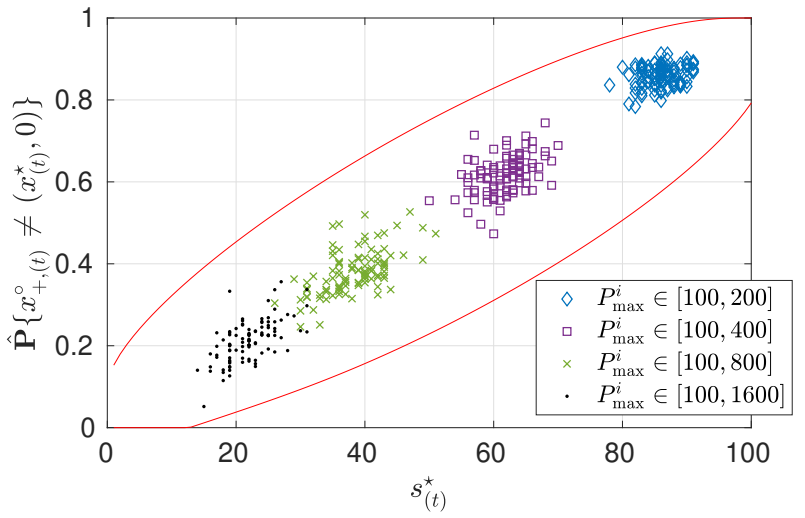

(a) $m=100$

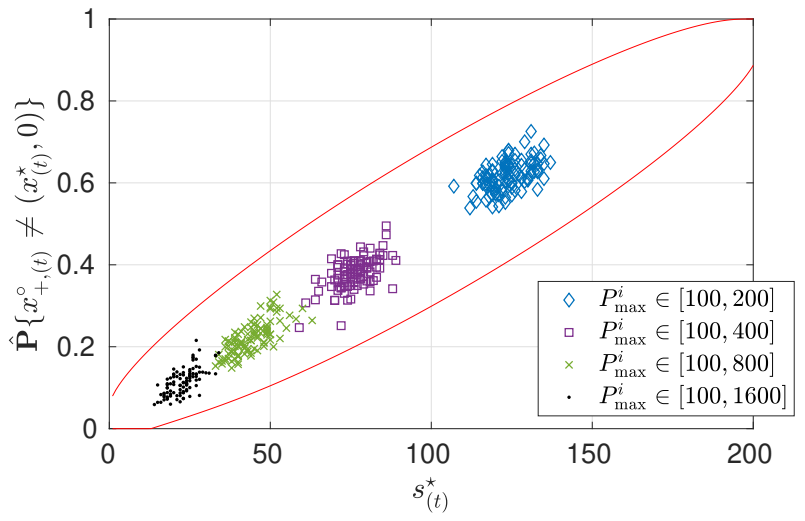

(b) $m=200$

Fig. 2. Simulation results for $m \in\{100,200\}$ and $P_{\max } \in\{200,400,800,1600\}$. Solid line shows the theoretical upper and lower bounds $\bar{\epsilon}(k), \underline{\epsilon}(k)$ on the probability that the optimal solution changes upon the arrival of a new agent. Each cloud of points corresponds to a different choice of $P_{\max }$ as indicated in the legend, and involves 100 points. Each point within a cloud corresponds to a different batch of $m$ energy producers and shows the empirical probability $\hat{\mathbb{P}}\left\{x_{+,(t)}^{\circ} \neq\left(x_{(t)}^{\star}, 0\right)\right\}$ ( $x$-axis) vs. the number of active agents ( $y$-axis).

[8] G. Notarstefano and F. Bullo, "Distributed abstract optimization via constraints consensus: Theory and applications," IEEE Transactions on Automatic Control, vol. 56, no. 10, pp. 2247-2261, 2011.

[9] M. Zhu and S. Martinez, "On distributed convex optimization under inequality and equality constraints," IEEE Transactions on Automatic Control, vol. 57, no. 1, pp. 151-164, 2012.

[10] M. Bürger, G. Notarstefano, F. Bullo, and F. Allgöwer, "A distributed simplex algorithm for degenerate linear programs and multi-agent assignments," Automatica, vol. 48, no. 9, pp. 2298 - 2304, 2012.

[11] A. Falsone, K. Margellos, S. Garatti, and M. Prandini, "Dual decomposition for multi-agent distributed optimization with coupling constraints," Automatica, vol. 84, pp. 149-158, 2017.

[12] K. Margellos, A. Falsone, S. Garatti, and M. Prandini, "Distributed constrained optimization and consensus in uncertain networks via proximal minimization," IEEE Transactions on Automatic Control, vol. 63, no. 5, pp. 1372-1387, 2018.

[13] A. Falsone, I. Notarnicola, G. Notarstefano, and M. Prandini, "Tracking-ADMM for distributed constraint-coupled optimization," Automatica, vol. 117, p. 108962, 2020.

[14] P. Gilmore and R. Gomory, "A linear programming approach to the cutting-stock problem," Operations Research, vol. 9, no. 6, p. 849-859, 1961.

[15] G. Owen, "On the core of linear production games," Mathematical Programming, vol. 9, no. 1, pp. 358-370, 1975.

[16] A. Falsone, K. Margellos, S. Garatti, and M. Prandini, "Linear programs for resource sharing among heterogeneous agents: the effect of random agent arrivals," IEEE Conference on Decision and Control, pp. 3853-3858, 2017.

[17] G. Calafiore and M. Campi, "The scenario approach to robust control

[23] M. Campi, S. Garatti, and F. Ramponi, "A general scenario theory for design," IEEE Transactions on Automatic Control, vol. 51, no. 5, pp. 742-753, 2006

[18] M. Campi and S. Garatti, "The exact feasibility of randomized solutions of uncertain convex programs," SIAM Journal on Optimization, vol. 19 , no. 3, pp. 1211-1230, 2008

[19] M. Campi, S. Garatti, and M. Prandini, "The scenario approach for systems and control design," Annual Reviews in Control, vol. 33, no. 2 , pp. $149-157,2009$.

[20] S. Garatti and M. Campi, "Risk and complexity scenario optimization," Mathematical Programming, pp. 4067-4078, 2019, publishe on-line. DOI: https://doi.org/10.1007/s10107-019-01446-4.

[21] L. Deori, S. Garatti, and M. Prandini, "A randomized relaxation method to ensure feasibility in stochastic control of linear systems subject to state and input constraints," Automatica, vol. 115, p. 108854 , 2020.

[22] M. Campi and S. Garatti, "Wait-and-judge scenario optimization," Mathematical Programming, vol. 167, pp. 155-189, 2018. nonconvex optimization and decision making," IEEE Transactions on Automatic Control, vol. 63, no. 12, pp. 4067-4078, 2018.

[24] A. Falsone, K. Margellos, J. Zizzo, M. Prandini, and S. Garatti, "On the sensitivity of linear resource sharing problems to the arrival of new agents," arXiv preprint, pp. 1-14, 2021. [Online]. Available: http://arxiv.org/abs/2109.13580

[25] D. Bertsimas and J. Tsitsiklis, Introduction to linear optimization. Athena Scientific Belmont, MA, 1997, vol. 6.

[26] M. Campi and S. Garatti, "Scenario optimization with relaxation: a new tool for design and application to machine learning problems," ArXiv, 2020, https://arxiv.org/abs/2004.05839. 